\title{
Simulated versus Hardware Laboratories for Control Education: A Critical Appraisal
}

\author{
James S. Welsh ${ }^{*}$ Talib Daredia ${ }^{* *}$ Frank Sobora* \\ Ljubo Vlacic ${ }^{* * *}$ Graham C. Goodwin* \\ * Centre for Complex Dynamic Systems and Control, \\ The University of Newcastle, Australia \\ (e-mail: james.welsh@newcastle.edu.au) \\ ** Matrikon Pty. Ltd. 15 Warabrook Boulevard, Warabrook, Australia \\ ${ }_{* * *}$ Institute for Integrated and Intelligent Systems, \\ Griffith University, Australia. (e-mail: l.vlacic@griffith.edu.au)
}

\begin{abstract}
The traditional approach to control education in Universities has been to enhance student learning with hardware style experiments. The associated experiments are always constrained by the fact that hardware must be provided. Thus typical experiments use tanks of water, servo motors, inverted pendula etc. These experiments are good in so far as they go. However, quoting a former student, "It is a bit like learning to fly a Jumbo Jet. One has the choice to learn on real hardware (say an ultralight aircraft) or on a simulator of the real aircraft under real flight scenarios". This paper explores this issue for control education and presents feedback from students comparing traditional hardware experiments with simulated experiments based around real world control system designs.
\end{abstract}

Keywords: control education

\section{INTRODUCTION}

There is international concern that Engineering Education is not responding to the demands of either end users (i.e. industry) or participants (i.e. students). Two of the most frequent messages are that engineering education needs to be more design orientated and more exciting for students. Similar comments apply to the specific topic of control engineering education. Indeed, there has been substantial international research conducted by both academia and industry which shows that the current first course in control in University is poorly understood and lacks motivation for students. (Dorato [1999], Bristol [1986], Murray et al. [2003], Bissell [1999], Dorato and Abdallah [1993], Antsaklis et al. [1999]).

In 1998, the U.S. National Science Foundation and the IEEE Control Systems Society hosted a workshop with a wide range of participants from university faculty, industry and government laboratories. A key conclusion was the strong need for curriculum reform in control education. The findings indicated that, in general, the current first course does not "adequately serve the future professional needs of students nor the need for practical control knowledge in an industrial setting." Moreover, the majority of students do not attempt to continue with any other control classes as a direct consequence. (Antsaklis et al. [1999]).

An industry practitioner Bristol [1986] states "if more engineers understood tuning of real processes and were not limited by the theoretical fables necessary in current theory of statistically derived models, then practical adaptive control would be further developed". Bissell [1999], remarks that evidence shows that current control education bears little value to students after graduation and what is learned is not well understood. Commenting on university graduate performance, an industrial manager notes with regret students currently come out as "parochial thinkers rather than systems engineers" (Bissell [1999]).

Dorato [1999] has remarked that graduate control education is much less of a problem, and the trick lies in retaining students at the undergraduate level. Currently, with students not being retained, control is being seen as a "service" discipline for the standard engineering programs. The worldwide undergraduate education concern is highlighted in a survey conducted on university faculty across twelve countries consisting of mostly electrical and control departments. One of its key findings was a "need to improve undergraduate laboratory experiences." (Dorato and Abdallah [1993]).

Key recommendations from both academia and industry to the findings outlined above consistently suggest the following new approach to undergraduate control education:

- Develop a wide range of new teaching and laboratory materials that instructors can select from to systematically introduce control techniques (Murray et al. [2003], Ashrafiuon and Bernstein [2005], Antsaklis et al. [1999]).

- Include proven modern real world theoretical results to supplement the classical trial-and-error theory currently taught. (Antsaklis et al. [1999], Bristol [1986], Murray et al. [2003], Zhu [1994]).

- Include hands-on experiments and design problems (Antsaklis et al. [1999]). 
- Incorporate further material on computer control, simulation and experimentation (Dorato [1999], Bristol [1986]).

- Demonstrate theoretical techniques experimentally showing both realistic success and failure (Bristol [1986]).

- Implement good and reasonably priced case studies and software for computer assisted instruction in control (Mansour and Schaufelberger [1989], Murray et al. [2003], Ashrafiuon and Bernstein [2005], Antsaklis et al. [1999]).

- Implement laboratories and software tools which demonstrate both theory and real life problems, provide visual and acoustic sensation, have a suitable time scale, be nonhazardous, inexpensive, and be easy to understand and use (Åström and Lundh [1992], Murray et al. [2003], Antsaklis et al. [1999]).

To prepare and excite students for the modern control world, laboratory learning is deemed necessary to complement traditional teaching material. It can exemplify sound engineering principles as well as demonstrate the corresponding real-life practicalities (Kocijan et al. [1997], Wellstead [1990], Åström and Lundh [1992]). Intentionally equipping laboratories with equipment having issues such as saturation, noise, sensing and actuation devices provides exposure to instrumentation and additional motivation to students (Wellstead [1990], Antsaklis et al. [1999]). An industry practitioner (Bristol [1986]) further verifies that "practice is introduced by laboratory experiences of varying vigor".

Laboratory examples demonstrating some of the above mentioned traits can be found in Pallini et al. [1998], Åström and Lundh [1992], Mansour and Schaufelberger [1989], Ko et al. [2001] and de Magistris [2005]. For example, Pallini et al. [1998] describe a virtual laboratory on wave propagation that allows students to experiment and observe results of their numerical simulations via animation techniques. This single laboratory is geared towards teaching highly mathematical and theoretical approaches. Åström and Lundh [1992] discuss the integrated use of software packages like Simnon and MATLAB with their purpose built physical laboratory processes. In a similar development Mansour and Schaufelberger [1989] describe a wide range of local physical laboratories integrated with software packages. However, as both of these are integrated with hardware components, they continue to bear the main disadvantages of physical laboratories.

In Ko et al. [2001], a virtual laboratory is described for a frequency modulation experiment that is accessed remotely via the internet. A MATLAB based virtual laboratory on quasi-stationary electromagnetics with a web interface for an electromagnetic course is discussed in de Magistris [2005]. Unfortunately, web based delivery laboratories come with their standard disadvantages such as bandwidth and browser compatibility concerns. The above examples are a select few of the growing number of undergraduate laboratories moving to the virtual concept.

Virtual experimentation has advantages over physical experimentation, especially when used in introductory courses. The first course in control typically has a large class size and thus there is a need for a large number of identical, reliable experiments. As virtual laboratories are software copies, by their nature they are remarkably reliable for every student. When the Lund Institute of Technology tried to implement existing physical commercial laboratories they were "quite disappointed both with respect to reliability and ergonomics" Åström and Lundh [1992]. On the other hand, a virtual laboratory has the advantage of being ergonomically feasible since it only requires the space of a computer monitor, a keyboard and a mouse. In many situations, it eliminates the need for lab space entirely. A virtual laboratory also bears the advantage of being relatively inexpensive when compared to physical laboratories. Furthermore, physical laboratory experiments are typically restricted by the timetable of the instructor, the students, the laboratory staff, and the laboratory room itself. A virtual laboratory eliminates these time constraints as the student may simply undertake the experiment at home or, indeed, anywhere. Moreover, a virtual laboratory has the key benefit of enabling students taking introductory level courses to tackle real world designs which is normally impossible in a typical teaching environment.

The use of multimedia technology and 'virtual reality' is widely advocated (Pallini et al. [1998]) as a significant way to improve the quality of engineering graduates. The interactive component allows the student to explore and observe, instantaneously, the effect of their own decisions, learning by trial and error. With regard to the software itself, applications built with a graphical user interface make for an effective learning tool rather than an additional learning task (Zhu [1994]). As discovered by the Control System Centre UMIST, in the United Kingdom, the ability to adjust parameters online is invaluable in building insight and judgemental ability in the student engineer (Wellstead [1990]). In the experience of the Swiss Federal Institute of Technology (ETH), a reasonable balance of teaching methods including computer usage helps students master a field known for its theoretical difficulties (Mansour and Schaufelberger [1989]).

The current paper contributes to the above circle of ideas by outlining student experiences gained from using a set of Virtual Laboratories developed at the University of Newcastle ${ }^{1}$ for both undergraduate and postgraduate students. The outline of the remainder of the paper is as follows: Section 2 reviews typical learning requirements in control education. Section 3 gives an overview of the Virtual Laboratories used in this study. Section 4 gives a more detailed overview of two laboratories to more clearly illustrate their scope. Section 5 summarises (both positive and negative) student feedback.

\section{THE LEARNING OBJECTIVES}

\subsection{First Course and Laboratory}

The current first year course in control typically covers theoretical aspects as compared to practical issues. On an international scale, utilizing recent syllabuses and information from the U.K. Bissell [1999], University of Newcastle [2007], Australia, University of Saskatchewan [2004],

\footnotetext{
1 For details see www.virtual-laboratories.com
} 
Canada, and the U.S. (Dorato [1999]), the following pattern of core material was found to be consistently taught:

- Linear systems theory (differential equations, frequency response, transfer functions)

- Classical single-loop design (Bode, Nyquist, and Nichols plots, frequency-domain design techniques, root locus)

The first course is normally limited to analog systems and in many cases is presented without any "hands-on" experience (Dorato [1999]). As found at the Lund Institute of Technology in Sweden, many students do not take the current special lab courses being offered as a complement to the theoretically oriented courses (Apkarian and Åström [2004]). Apkarian and Åström [2004] comment that "this reluctance is unfortunate because good experiments can also be a strong motivation to pursue a career in control". The typical setup for their control experiments consisted of a physical process with sensors, actuators, power supply and a PC equipped with interfaces. Since the time available for experiments in undergraduate courses is limited, it was found essential to avoid tedious hand calculations and programming (Åström and Lundh [1992]).

In most universities the introductory course has a large class size so it is necessary to have a large number of identical experiments. In 1990, a national survey of Electrical Engineering departments in the United States was conducted with 143 Universities responding. It found that almost $75 \%$ require at least one undergraduate control course, and a majority of these required a laboratory (Feliachi [1994]). According to the findings of the NSF/CSS workshop, to match its suggested goals, the control course "necessitates full integration of software and visual interactive material into the course" (Antsaklis et al. [1999]).

\subsection{Modern Control}

With theory and an understanding of practical control problems, a graduating Electrical Engineer should have the skills to tackle modern control issues. A typical modern control syllabus covers modelling, optimisation, constraint handling, full automation, data acquisition, implementation, control design and simulation. It utilizes high technology solutions, smart sensors/actuators/controllers, as well as flexible and highly configurable platforms with huge computational capacity (Apkarian and Åström [2004], Bernstein [1999], Crisafulli [2007]).

The Panel of Future Directions in Control, Dynamics and Systems (Murray et al. [2003]) consisting of participants from academia, industry and government developed a spectrum of examples of modern control and the associated implications of feedback. High level examples include robust networks linking businesses, transportation systems, energy infrastructure, as well as low level examples such as regulation and control of the electrical power grid, command and control of unmanned vehicles, and highaccuracy positioning of read/write heads in disk drives.

\section{THE UNIVERSITY OF NEWCASTLE VIRTUAL LABORATORY FOR CONTROL SYSTEMS DESIGN}

This paper summarizes student experiences gained with a particular set of tools namely the Virtual Laboratories for Control Systems Design (VLCSD) Laboratory. This is a stand alone software learning tool consisting of 17 modules. Each module has been designed to:

- review specific control principles

- provide an interactive problem based learning environment demonstrating real world practicalities

Students can self-pace their way through the experimentation process. Immediate feedback is given imparting the feeling of real world exposure.

The modules include the animated replication of standard university laboratories, real world designs, and novel experiments based on modern "high technology" devices.

A total of 17 modules is currently available as part of the VLCSD product as follows:

(1) Electromechanical Servomechanism

(2) Resonant Electromechanical Servomechanism

(3) Coupled Tanks

(4) Continuous Caster - Classical Control Design

(5) Continuous Caster - Nonlinear Issues

(6) Rolling Mill - System Modelling and Classical Control

(7) Rolling Mill - Soft Sensors

(8) Rolling Mill - Periodic Disturbances and Observer Design

(9) Rolling Mill - Kalman Filtering

(10) Rocket Dynamics

(11) Rocket Controller Design

(12) Cross Directional Control of Web Forming Processes - Interaction and Simple PID Control

(13) Cross Directional Control of Web Forming Processes - Actuator Saturation

(14) Cross Directional Control of Web Forming Processes - Robustness

(15) Audio Quantisation - Optimal Noise Shaping Quantiser

(16) Audio Quantisation - Bode Sensitivity Integrals

(17) Electromechanical Servomechanism - Affine Parameterisation

Laboratories 4, 5, 6, 7, 8, 9, 12, 13 and 14 refer to real world designs that have been fully implemented. Laboratories 15 and 16 are aimed at showing students how feedback ideas are used in high technology products e.g. CD mastering. Laboratories 1, 2, 3 and 17 are intended to provide a low cost alternative to typical University hardware experiments. Laboratories 10 and 11 take students into rocket dynamics and control. The learning objective of each laboratory is different but, as a whole, they cover the requirements outlined in section 2 .

\section{TYPICAL MODULES}

This section reviews two of the modules available in the VLCSD product to indicate the scope of the laboratories.

\subsection{Continuous Caster (Laboratory (4) and (5))}

This virtual laboratory is modelled on a real world design implemented on the BHP Continuous Caster in Newcastle, Australia. The laboratory gives the student an exposure to real world events that are difficult, and arguably impossible, to replicate in traditional physical laboratories. A 
photograph of a section of the real steel caster is shown in figure 1 .

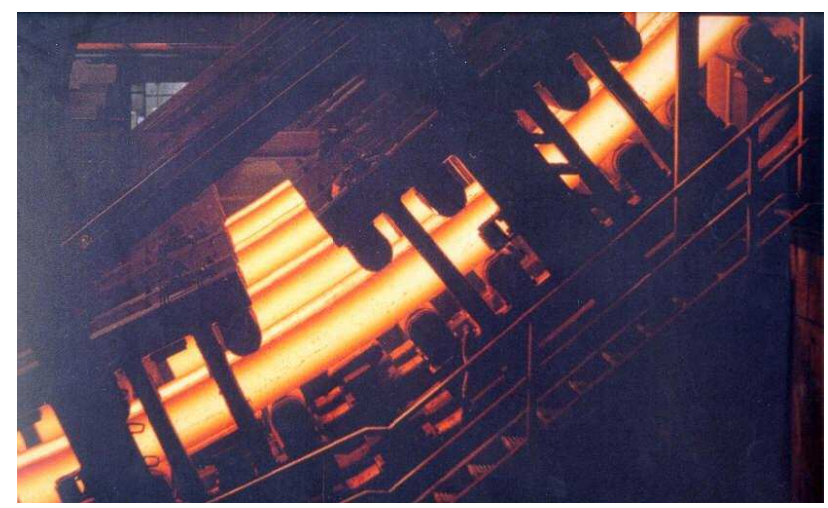

Fig. 1. Continuous Steel Caster

A Continuous Caster is a common means by which molten liquid steel is solidified. It comprises a reservoir of steel (the Tundish) directly above the mould as shown in figure 2. The flow of the molten steel is controlled by a Slide Gate Valve (SGV). An important issue with the continuous casting of the molten steel is that the level of steel in the mould be kept constant to a high degree of accuracy. If the level of steel is allowed to fluctuate too widely then contamination of the steel can occur. The contamination is capable of causing structural weakness in the resulting products. The liquid steel enters the mould via a submerged entry nozzle. The nozzle has an outer refractory lining to deal with the extreme conditions, however the flux material on the surface of the molten steel eventually destroys this lining. In order to maximize the nozzle life the mould level set point is periodically changed.

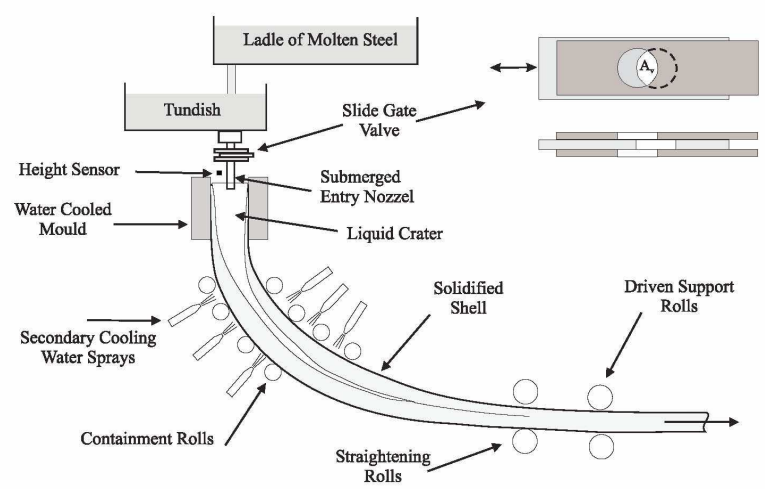

Fig. 2. Continuous Caster Schematic

In the Virtual Laboratory the students interactively experiment with the process via an animated software program. A screenshot of the Continuous Caster laboratory is shown in Figure 3.

A key issue in the design is that plant disturbances must be well accounted for by the controller using information provided by the plant instrumentation devices. During their experimentation, students may well see the plant go unstable and observe the corresponding costly, dangerous results. This type of scenario is crucial in the real world but usually not covered in typical University control education. The laboratory also covers many real world issues includ-

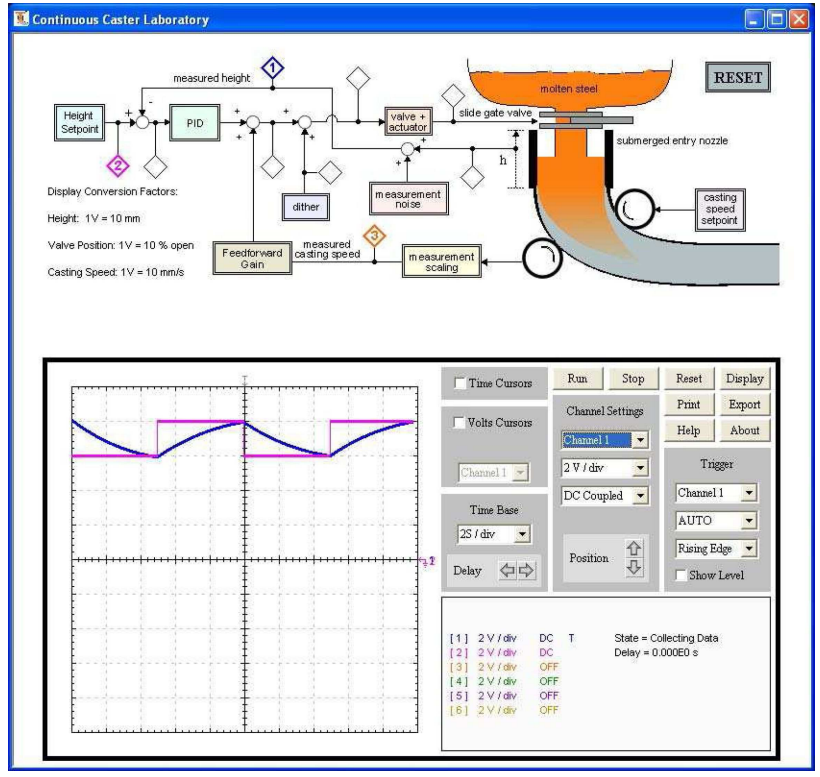

Fig. 3. Screenshot of the Continuous Caster Laboratory

ing sensor and actuator imperfections which are crucial in this application.

\subsection{Audio Quantisation (Laboratory (15) and (16))}

This laboratory shows that control system design plays a central role in modern high technology equipment, such as CD mastering. Here signal re-quantisation is a core issue. Re-quantisation corresponds to lowering the resolution of a finely quantised signal in order to provide a coarser quantised signal. In the CD mastering application, each sample is typically quantised to 8 bits, based upon master recordings of higher resolution (e.g. 16 bit).

Quantisation unavoidably introduces loss of information. In the case of audio signals, the challenge is to preserve the perceived sound quality as much as possible. The aim in the mastering process is to render the difference between the original and the quantised signal, inaudible to the listener. In view of the importance of the perceived sound quality, significant research effort has been concentrated into the development of psychoacoustically optimal quantisers. The simplest scheme of this type uses feedback to shape the frequency content of the quantization errors as perceived at the output - see figure 4 .

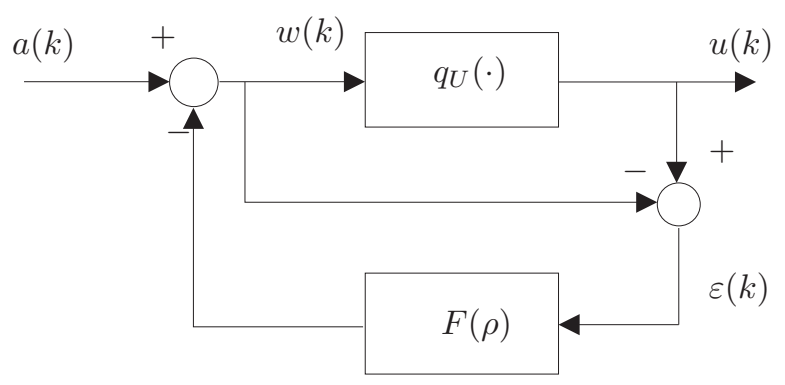

Fig. 4. Noise Shaping Quantiser

This laboratory illustrates the 'Power of Feedback' in a simple and easily understood example. The users of this 
laboratory receive both visual and acoustic stimulus and thus 'hear' the difference that feedback makes. A view of the user interface for part of the audio laboratory is shown in figure 5. The learning objectives include:

- the psychoacoustic properties of human hearing

- the effect of quantisation error feedback on the performance of a quantiser

- feedback analysis of noise shaping quantisation

The second laboratory in this pair covers Bode integrals and illustrates sensitivity trade-offs and performance limitations.

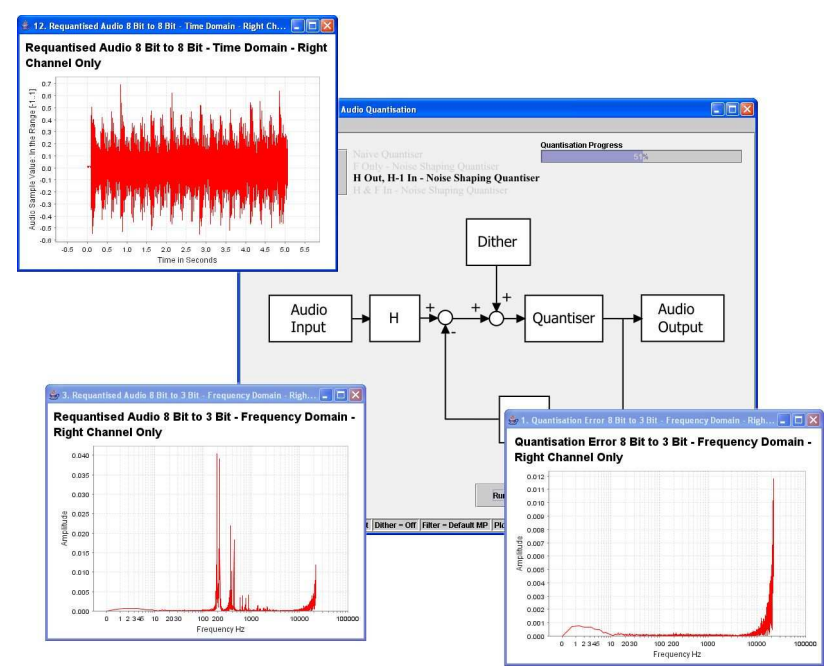

Fig. 5. Screenshot of the Audio Laboratory

\section{STUDENT FEEDBACK}

We have evaluated the full set of Virtual Laboratories with both undergraduate students and recent graduates in industry. Some of the comments are summarized below. (Note: We have included both positive and negative comments.)

- It is a bit like learning to fly a jumbo jet. One has the choice to learn on real hardware (say an ultralight aircraft) or on a simulator of the real aircraft under realistic flight scenarios. These laboratories fall into the latter category.

- The worst aspect of the virtual laboratory was not being able to experience the practical aspects of control, such as wiring, data acquisition, sensor and actuator calibration, etc. It was however a relief not having to perform these things, especially when I completed the lab over several sittings and having to re-calibrate the equipment wasn't necessary.

- You are able to do this at your own time and at home. The process is also faster than the real system.

- The results obtained were consistent and accurate. A problem with the 'real' hardware labs is that results may vary between apparatus and results obtained one day may be different to the next.

- It was good to be able to do it in my own time at home (especially good for distance education).

- From a student perspective I can see that a necessary evil of the laboratories would be the background theory required. However all background questions in this lab were relevant to the current stage of the control system design and were able to be quickly verified on the program.

- I have already completed the hardware version of this laboratory (coupled tanks); however I think the the virtual computerized laboratory makes it a different experience. I think you would achieve a similar educational experience from both. With the virtual laboratory you benefit in computer skills using the apparatus in the program such as the oscilloscope. However you do not get the hands on approach that you would achieve from using the real system.

- The laboratories allow one to do the work at home and you wouldn't have to do it at a certain time like you would with an on-campus laboratory.

- The "virtual" laboratory concept definitely has its pros and cons. The speed and the fact that you can do it when you want are good but it has downside like no hands on experience or if you have difficulty no lab demonstrator. Overall I think it is a good experience and its pros out way its cons.

- The laboratory develops key control theories that are used in industry. Also in industry a lot of engineering is done using computers and simulation with computers so it is beneficial in that way.

- I found that not having to worry about the practical side of things (such as wiring and data acquisition) provided a better education experience in terms control theory. I also think that those practical things are important aspects of control and can't be forgotten about all together. In short, I think that the simulation lab was a better educational tool in terms of the theory of control and would help to provide a stronger knowledge base than the usual "toy" systems would.

- I liked the freedom it provides (where and when I can do it) and being able to purely focus on the control aspects of the lab.

- I believe that virtual laboratories can provide a more specific learning experience by removing all of the problems associated with 'real systems'. I also believe however that it should be used to complement physical laboratories and not replace them all together.

- I believe that the concepts in the laboratory are very transferable to a practical system however many aspects (such as wiring, data acquisition, etc) of true industrial systems were missing. The virtual laboratory is an excellent tool to educate students about control on industrial type systems.

- Advantages:

Allows you to focus more on control aspects and hence becomes a better educational experience.

- Easier to perform seeing as time isn't wasted on setting up equipment and learning how to use data acquisition software, etc.

- Results are known to be accurate.

- Can do the lab at home in your own time.

- Cost of the lab is probably less and there is no maintenance required on equipment.

Disadvantages:

- You don't get to experience the physical aspects of control.

- Less chance that students will work as a group. 
- I felt like the laboratory stepped through the control process in a logical way, starting from creating a model of the plant and then applying different control strategies. This is similar to the coursework structure.

- As a recent graduate now working as a process control engineer, I found it very beneficial to be able to experiment and test different control strategies on processes that are very typical in industry. On a working plant it would be very rare to have the freedom to experiment with and test new control strategies.

- The virtual laboratory certainly allows students to have an exposure to a much wider range of challenging systems. The gearing towards typical processes found in today's industry can only benefit the developing control student. I feel these labs provide better educational experience as they still introduce the various control tools of conventional labs with the added benefit of industrial awareness.

- Engineering students are faced not only with large academic work loads but external work and personal commitments. The flexibility provided by virtual laboratories would thus be appreciated by students.

- The ability to start and stop the laboratory is a nice feature. You can easily pick up from where you left off in a matter of seconds. Rather then have to physical set up and recalibrate lab. This makes it ideal if you only have short window to work on the lab. Another appealing feature is their portability, you can work on campus or easily take home to complete.

- I completely endorse the virtual laboratory concept. I agree that these laboratories should not replace exposure to physical labs but act as a supplement to these experiences. The concept provides many additional features such as portability (can be done anywhere, anytime), straight into understanding real world process and testing control strategies.

- By introducing aspects such as nonlinear valves and slip stick phenomena, the control problem is very practical and typical to challenges faced by control engineers in industry.

- The idea of solving industry problems is a very attractive feature of the virtual lab it gives students a sense of real relevance that are not always possible with toy systems.

- This lab definitely complements the learning I received while at university. It covered aspects and challenges very typical to an industrial process.

- I think the ability to contrast the effects that nonlinear or sticky valves have on the control system with the flick of a switch really allows the student to appreciate their effect. This may be clouded on standard physical labs.

- Probably the most important aspect of the virtual laboratory is the range of real industrial control problems that can be undertaken. This certainly helps to generate interest and a feeling of purpose in what you are doing not just reading values on an oscilloscope.

\section{CONCLUSIONS}

Students and practicing engineers have generally welcomed the virtual laboratory concept. The most common positive comments are the ability to have exposure to real world designs and the flexibility of working at one's own time and place. Negative comments included the fact that one does not get exposure to wiring, calibration, interfacing etc. However, to some extent, this was off-set by speed and repeatability. Overall we believe that the reaction to the virtual laboratory concept is very positive and we hope others may be persuaded to try this method of giving real world experiences to students in a University environment.

\section{REFERENCES}

P. Antsaklis, T. Başar, R. DeCarlo, N.H. McClamroch, M. Spong, and S. Yurkovich. Report on the nsf/css workshop on new ddirections in control engineering education. IEEE Control Systems Magazine, 19(5):53-58, October 1999.

J. Apkarian and K. Åström. A laptop servo for control education. IEEE Control Systems Magazine, 24(5):70-73, October 2004.

H. Ashrafiuon and D.S. Bernstein. Innovations in undergraduate education: Part ii. IEEE Control Systems Magazine, 25(1):21-22, February 2005.

K. Åström and M. Lundh. Lund control program combines theory with hands-on experience. IEEE Control System Magazine, 12 (3):22-30, June 1992.

D.S. Bernstein. Enhancing undergraduate control education. IEEE Control Systems Magazine, 19(5):40-43, October 1999.

C.C. Bissell. Control education: Time for radical change? IEEE Control Systems Magazine, 19(5):44-49, October 1999.

E.H. Bristol. An industrial point of view on control teaching and theory. IEEE Control Systems Magazine, 6(1):24-27, February 1986.

S. Crisafulli. Managing Director, Matrikon Pty. Ltd, PO Box 516, Mayfield, NSW, Australia, 2007.

M. de Magistris. A matlab-based virtual laboratory for teaching introductory quasi-stationary electromagnetics. IEEE Transactions on Education, 48(1):81-88, 2005.

P. Dorato. Undergraduate control education in the us. IEEE Control Systems Magazine, 19(5):38-39, October 1999.

P. Dorato and C. Abdallah. A survey of engineering education outside the united states: Implications for the ideal engineering program. Journal of Engineering Education, 82(4):212-215, 1993.

A. Feliachi. Control systems curriculum national survey. IEEE Transactions on Education, 37(3):257-263, 1994.

C.C. Ko, B.M. Chen, S. Hu, V. Ramakrishnan, C.D. Cheng, Y. Zhuang, and J. Chen. A web-based virtual laboratory on a frequency modulation experiment. IEEE Transactions Systems, Man, and Cybernetics, Part C: Applications and Reviews, 31(3): 295-303, 2001.

J. Kocijan, J. O'Reilly, and W.E. Leithead. An integrated undergraduate teaching laboratory approach to multivariable control. IEEE Transactions on Education, 40(4):266-272, 1997.

M. Mansour and W. Schaufelberger. Software and laboratory experiments using computers in control education. IEEE Control Systems Magazine, 9(3):19-24, April 1989.

R.M. Murray, K. Åström, S.P. Boyd, R.W. Brockett, and G. Stein. Future didrections in control in an information-rich world. IEEE Control Systems Magazine, 23(2):20-33, April 2003.

University of Newcastle. Elec4410 course syllabus. Australia, 2007. University of Saskatchewan. Ee 481 course syllabus. Canada, 2004.

L. Pallini, S. Piroli, L. Stacchio, and F. Zirilli. Computer-aided instruction in wave propagation phenomena. IEEE Engineering Science and Education Journal, 7(5):215-220, 1998.

P.E. Wellstead. Teaching control with laboratory scale models. IEEE Transactions on Education, 33(3):285-290, 1990.

J.J. Zhu. Motivation-by-challenge for teaching control systems. IEEE Control System Magazine, 14(5):64-68, 1994. 